\title{
Responsabilidad social: ¿un regalo o un gasto necesario? Entre el buen gobierno corporativo y la determinación del impuesto a la renta Social responsibility: A gift or a necessary expense? Between good corporate governance and the determination of income tax
}

\author{
MÓNICA BENITES MENDOZA*
}

Resumen: El presente artículo pretende aportar, mediante el debate, al tema de los gastos relacionados con la responsabilidad social, materia que ha tomado relevancia últimamente. En este artículo lo que pretendemos es observar hasta qué punto este tipo de gastos cumple con el criterio de causalidad, requisito necesario para que sea deducible.

Palabras clave: responsabilidad social - gastos deducibles

Abstract: This paper seeks to contribute, through discussion, to the issue of costs related to social responsibility, a matter that has recently gained importance. In that sense, our intention is to examine to what extent such costs meet the criterion of causation, a necessary requirement to be deductible.

Key words: social responsibility - deductible expenses

CONTENIDO: I. INTRODUCCIÓN.- II. DESARROLLO.- III. CONCLUSIONES.

\section{INTRODUCCIÓN}

En los últimos años, hemos observado que muchas empresas adoptan al interior de sus organizaciones el desarrollo y aplicación de los principios de un buen gobierno corporativo. Dentro de esta concepción, la responsabilidad social es un pilar y en muchos casos es necesario contar con este tipo de programas de ayuda para obtener certificaciones internacionales de calidad. No es suficiente acreditar la eficiencia como empresa, sino que también es necesario demostrar que efectivamente existe una armonía con el ambiente que nos rodea.

Por ello resulta pertinente preguntarnos si el gasto por responsabilidad social es deducible, si nuestra normativa o jurisprudencia ha establecido

* Abogada por la Pontifica Universidad Católica del Perú, con estudios concluidos en la Maestría de Derecho Constitucional y con una licenciatura en Educación. Socia del Estudio Benites \& Abogados. Correo electrónico: benites@ benitesabogados.com.pe 
algún criterio y cuál es la diferencia con una liberalidad. Todas estas dudas surgen al tratar este tema de tan actual relevancia, es así que en el presente artículo pretendemos brindar una evaluación acerca de este tipo de gastos y colaborar en establecer si puede observarse una diferencia entre este gasto necesario y la liberalidad, ya que la línea que los separa parece ser muy tenue.

\section{DESARROLLO}

Una primera aproximación sería señalar que en este tipo de gastos acreditarse debidamente no solo la necesidad de realizarlos, sino que debe estar sustentada la aplicación del principio de causalidad. Dicho principio genera e inicia la controversia respecto a determinar desde qué perspectiva entendemos que un gasto es causal; es decir, cómo establecemos el marco legal regulatorio del alcance y límites de la aplicación de este principio, si desde el punto de vista exclusivamente de la empresa o si desde la perspectiva de una aplicación integradora de empresa y entorno.

En este orden de ideas, la pregunta por cómo se aplica el principio de causalidad para gastos de esta naturaleza refiere implícitamente a si el marco tributario va a propender a la búsqueda de un equilibrio entre las partes ya señaladas, bajo la perspectiva de un desarrollo no solo lucrativo para la empresa (entendido como tal solo aquel que propende a incentivar la generación y/o mantenimiento de la renta), sino armonioso con la realidad social que lo rodea (que propende no solo a evitar los conflictos sociales que puedan paralizar la fuente productora de renta, sino que también coadyuva a un desarrollo equilibrado del entorno social que retroalimente y otorgue adecuado soporte a esta indicada fuente).

Cabe citar entonces a Carbal Herrera, quien indica que «[...] bajo estas circunstancias [la perspectiva empresarial] invertir una parte de las utilidades de las empresas en actividades en pro de la preservación del medio ambiente y del mejoramiento de la calidad de vida de los diversos grupos de interés, resultan improcedentes». Dentro de esta perspectiva, señala que «[...] reconoce solo el camino del desarrollo económico y desconoce horizontes sociales y ambiéntales que se abren a su paso» ${ }^{1}$.

Entonces la controversia parece residir no únicamente en si aplicamos adecuadamente el principio de causalidad, sino en cómo entendemos el citado principio de causalidad. La pregunta a plantearse entonces sería, desde qué perspectiva analizamos el concepto técnico de causalidad, idesde la óptica empresarial (que implica beneficio directo o indirecto

1 Carbal Herrera, Adolfo. Responsabilidad social y contabilidad: apuntes críticos. Bogotá: Universidad Libre de Colombia, 2009, p. 88. 
para la empresa exclusivamente) o desde la óptica integradora con lo social y medioambiente, en su relación directa con la empresa? De acuerdo con lo anteriormente indicado, el principio de causalidad deberá entenderse o como lo exclusivamente necesario para la empresa o como lo estrictamente requerido adicionalmente para el entorno en el que se desenvuelve.

Para determinar el alcance de lo inicialmente planteado, empezaremos por revisar lo ya trabajado a nivel de doctrina y jurisprudencia. Es así que sobre este tipo de gastos, nuestro máximo intérprete - la Constituciónha establecido algunos criterios que sustentan la necesidad de realizarlos. Al respecto, Arroyo Langshwager menciona, algunos pronunciamientos emitidos por el Tribunal Constitucional. A continuación detallamos dichos pronunciamientos:

\section{Pleno Jurisdiccional 0048-2004-PI-TC:}

Ser socialmente responsable no significa solamente cumplir plenamente las obligaciones jurídicas, sino también ir más allá del cumplimiento invirtiendo en el entorno local y contribuyendo al desarrollo de las comunidades en que se inserta, sobre todo de las comunidades locales. En el marco del Estado Social y Democrático de Derecho de la Economía Social de Mercado y del Desarrollo Sostenible, la responsabilidad social se constituye en una conducta exigible a las empresas, de forma ineludible?

De lo indicado se observa que, de acuerdo con el criterio señalado por el Tribunal Constitucional, existe en las empresas una conducta o un deber ineludible de ser responsables socialmente, por lo que si existe ello, para viabilizar esa conducta la empresa debe realizar gastos, los cuales serán perfectamente deducibles, bajo dicho presupuesto, por cumplir con el criterio de causalidad. Otro pronunciamiento que apoya esta necesidad de realizar estos gastos relacionados con la responsabilidad social, lo observamos en la sentencia que resuelve el expediente 03343-2007-PA/TC: «En el marco del Estado Social y Democrático de Derecho de la Economía Social de Mercado y del Desarrollo Sostenible, la responsabilidad social se constituye en una conducta exigible a las que ineludiblemente corresponde a la empresa».

En la actualidad, existe consenso en indicar que la actividad empresarial siendo esencialmente lucrativa, no se opone a que asuma su responsabilidad social. Los efectos que las empresas generan han suscitado que se tomen ciertas medidas a fin de lograr una inserción mas pacífica de la empresa en la sociedad. Es así como se ha desarrollado el concepto de responsabilidad social de la empresa, que tiene diversos ámbitos de

2 Arroyo Langshwager, Gisela. «La deducción del Impuesto a la Renta de los Denominados "Gastos de Responsabilidad Social" efectuados en etapas preproductivas". Revista IPDT 53 (2013), pp. 107-136, pp. 108-109. 
aplicación como el interno: el relativo al respecto de los derechos laborales de los trabajadores y al clima laboral interno, así como al buen gobierno corporativo; $y$ en el externo, que enfatiza más las relaciones entre la empresa y la comunidad y su entorno ${ }^{3}$.

En este caso, el Tribunal Constitucional nos da una directriz al precisar que este tipo de gastos no solo sirven para evitar el conflicto, sino que en muchas ocasiones se utilizan como medios para que la empresa pueda desempeñarse de una manera óptima. Es así que coincidimos con las conclusiones de la autora al señalar lo siguiente:

De lo antes señalado podemos considerar como características de la responsabilidad social empresarial a las siguientes:

- La responsabilidad social forma parte de una estrategia empresarial y constituye una visión de negocios cuyo objetivo es incrementar la rentabilidad de las empresas y garantizar el desarrollo de sus proyectos en armonía con la comunidad el Estado.

- Los gastos por concepto de responsabilidad social constituyen herramientas necesarias para mejorar la competitividad y sostenibilidad de las empresas, permiten un ambiente adecuado para el desarrollo de las actividades generadores de renta y la continuidad de las actividades empresariales.

- La responsabilidad social es una conducta exigible a las empresas de rango construccional ${ }^{4}$.

Como puede observarse, este tipo de gastos, en esta época, resultan necesarios para el desempeño de las empresas porque con la realización de estas erogaciones se conseguirán otros fines y se ayudará a un mejor desenvolvimiento con el entorno que le rodea.

Se trata entonces de gastos que son complementarios al desenvolvimiento del giro de la empresa. Asimismo, es importante precisar que estos deben distinguirse de las denominadas liberalidades, las cuales, como se sabe, nunca constituirán un gasto deducible, porque nuestra norma tributaria es clara y explícita al señalar que solo son deducibles los gastos causales.

En ese orden de ideas, cabe citar a Santiváñez Guarniz, quien indica lo siguiente:

Dentro de la normativa internacional, no se conoce alguna norma contable, económica o financiera que regule qué gastos son necesarios o no para los negocios, salvo el principio de asociación que vincula el gasto relacionado con un ingreso (conocido como principio de causalidad). [...] La determinación, para efectos tributarios, con exactitud, de la 
causalidad entre ingresos y gastos es una tarea, en muchos casos, muy compleja y, a nuestra entender, deben tomarse en cuenta aspectos principales del negocio como plan estratégico, objetivos y metas a corto y largo plazo, estrategias de posicionamiento, procesos internos y estrategias en relación con el personal con la finalidad de determinar, al menos en forma razonable la mencionada causalidad ${ }^{5}$.

Es importante recordar que existe abundante doctrina que señala que el principio de causalidad que los gastos deben cumplir no puede ser interpretado de manera restrictiva, porque la lista de gastos recogida en el artículo 37 de nuestra Ley del Impuesto a la Renta no es una lista cerrada. Es así que Alva Matteucci nos recuerda lo siguiente sobre este tema:

En realidad, al efectuar una lectura más a fondo sobre el tema (aplicando otros métodos de interpretación como el sistemático o el teleológico) apreciamos que para poder determinar si un gasto es deducible o no, es necesario identificar que este sea necesario para la generación de la renta y/o el mantenimiento de la fuente productora de la renta y no una simple lectura de los literales del artículo $37^{\circ}$, sino que se debe estudiar el encabezado del propio artículo. Ello significa que se estaría aplicando una lista abierta, que en latín se le conoce como numerus apertus. En este orden de ideas, apreciamos que el concepto que la LIR contempla con respecto al principio de causalidad no es restringido sino amplio, con lo cual constituye una lista abierta y se adopta entonces la concepción amplia que se abordó en el punto anterior ${ }^{6}$.

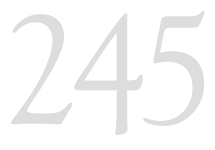

RESPONSABILIDAD SOCIAL: ¿UN

REGALO O

UN GASTO

NECESARIO?

ENTRE EL BUEN

GOBIERNO

CORPORATIVOY LA

DETERMINACIÓN

DEL IMPUESTO A

LA RENTA

SOCIAL

RESPONSIBILITY:

A GIFT OR A

NECESSARY

EXPENSE?

BETWEEN GOOD

CORPORATE

GOVERNANCE

AND THE

DETERMINATION

OF INCOME TAX

Asimismo el autor Durán Rojo precisa, sobre la causalidad del gasto, que debe considerarse tanto el plano objetivo como el subjetivo, este último plano es el de aquello que responde a una decisión gerencial, es allí donde se encontrarían este tipo de gasto. Así, el autor precisa lo siguiente:

Ahora bien, la causalidad del gasto — para producir rentas gravadas o mantener su fuente- puede ser entendida en función de distintas clasificaciones. Así, puede hablarse de un plano objetivo y otro subjetivo de la causalidad del gasto. En el primer caso, tendremos aquellos gastos que hay que sufragar con carácter obligatorio o de manera indispensable (como son los establecidos por ley o los que corresponden a servicios públicos, por ejemplo) y, en el segundo, podríamos encontrar a aquellos que provienen de decisiones de la gestión empresarial. La acreditación

5 SANTIVÁÑEZ GUARNIZ, Juan. «Sobre la causalidad de los gastos deducibles para efectos del Impuesto a la Renta. Un enfoque empresarial». Contabilidad y Negocios, 4, 7 (2009), pp. 17-22, pp. 21-22.

6 Alva MATTEUCCI, Mario. «El principio de causalidad y su implicancia en el sustento de los gastos en el Impuesto a la Renta». Actualidad Empresarial, 197 (2009), pp. I1-I3, p. I2. 
de la fehaciencia del gasto será mayor cuando el egreso se encuentre más en un plano subjetivo que en el plano objetivo?

Por ello y considerando la amplitud del concepto de causalidad, de acuerdo con lo anteriormente indicado (beneficio no solo directo, sino indirecto, inmediato o mediato), es pertinente preguntarnos si estos gastos de responsabilidad social son liberalidades. De acuerdo con nuestra jurisprudencia y doctrina, estos gastos de responsabilidad social no pueden ser catalogados como liberalidades, ya que no son otorgados porque la empresa tiene un acto de desprendimiento, sino que los realiza con la finalidad, por ejemplo, de evitar un conflicto, de solucionar un problema que permita generar mayor renta o mantener la fuente de trabajo, mejorar las relaciones con las comunidades que rodean a la empresa, una obra que permita que la empresa pueda obtener mayores rentas o dar un mejor mantenimiento a la fuente productora de renta.

En ese orden de ideas, el Tribunal Fiscal, en distinta jurisprudencia, ha definido la liberalidad como «el desprendimiento, la generosidad, la virtual moral que consiste en distribuir uno generosamente sus bienes sin esperar recompensa ${ }^{8}$. Es así que para el Tribunal Fiscal, cuando una empresa que realiza actividad empresarial entrega un bien o servicio sin esperar nada a cambio, se trata de una liberalidad. Entonces, en el caso de las erogaciones relacionadas con el gasto de responsabilidad social, es sostenible que las empresas lo realizan porque esperan algo a cambio, lo cual no necesariamente es directo, por lo que este tipo de gastos, de acuerdo con el criterio expuesto del Tribunal Fiscal, no podría ser catalogado como liberalidad.

Al respecto, cabe precisar que en nuestra jurisprudencia pueden encontrarse los siguientes ejemplos, donde el Tribunal Fiscal ha aceptado este tipo de gastos con algunos criterios rígidos para determinar la deducibilidad. En la resolución del Tribunal Fiscal 01424-5-2005 se señala lo siguiente:

Se reconoció la causalidad de los gastos de responsabilidad social, en un supuesto en que la recurrente había comprado bienes con el objeto de conservar el medio ambiente en la comunidad de Caravelí, aduciendo que la adquisición fue realizada para contrarrestar la contaminación a través de la reforestación y preparación de tierras para el sembrío de plantas en beneficio de los trabajadores de la mina y de las personas que viven alrededor de ella. Empero, como no se presentó documento alguno u otra prueba que demostrara la utilización de los bienes para los fines mencionados, se mantuvo el reparo?.

7 DuRÁN RoJo, Luis. «Alcances del principio de causalidad en el Impuesto a la Renta Empresarial». Contabilidad y Negocios, 47 (2009), pp. 5-16, p. 15. Las cursivas de la primera oración son nuestras. 8 Resoluciones del Tribunal Fiscal 02675-5-2007 y 09478-1-2013.

9 Las cursivas son nuestras. 
Aquí se puede observar que el beneficio directo lo tiene la comunidad, pero no puede negarse que la armonía con el medio ambiente y con esta comunidad aledaña permitirá que la empresa pueda mantener la fuente y hasta inclusive que genere mayor renta, al realizar este tipo de gasto. En estos casos, al estar relacionadas las erogaciones de este tipo con la actividad minera o extractiva, ellas sirven para eliminar los conflictos o suprimir los focos de violencia que puedan impedir el normal desarrollo de la empresa.

En el caso de la resolución del Tribunal Fiscal 016591-3-2010, el Tribunal reconoció el gasto, ya que se demostró que sirvió para evitar el conflicto social en la zona. El máximo órgano indicó lo siguiente:

Se aceptó la deducción de gastos incurridos en la entrega de bienes y prestación de servicios a los pobladores de las comunidades nativas de las áreas en las que la recurrente realizaba sus actividades. Se interpretó que dichos gastos no respondieron a una simple liberalidad, sino que por el contrario fueron realizados a fin de evitar conflictos sociales que directamente pudieran afectar el normal funcionamiento del yacimiento y demás instalaciones de la recurrente.

De lo expuesto, fluye que en estos casos, si bien se evidencia una línea tenue, existen ciertos gastos de responsabilidad social que son gastos deducibles (lo cual perfectamente puede sustentarse). Estos gastos no necesariamente están relacionados con la generosidad de las empresas, sino que estas esperan una contraprestación: un mejor ambiente en donde desenvolverse, evitar conflictos, mejorar la zona, situaciones todas que permiten que su actividad se realice de mejor manera y hasta disminuyendo algunos costos.

Es importante precisar al respecto, que si bien es cierto que la responsabilidad social se ha relacionado íntimamente con la actividad minera, ello solo constituye un ejemplo de las actividades en donde se puede aplicar este concepto. No por el hecho de que el concepto siempre haya estado relacionado con este tipo de actividades, debe este confundirse ni restringirse a ellas. Por ejemplo, no se podrá negar la causalidad del gasto que una empresa realiza en una hidroeléctrica para repararla y que pueda proveer de luz eléctrica al poblado, incluida la empresa, o debe de cuestionarse la causalidad del gasto porque no favorece de manera exclusiva a la empresa. En este caso consideraríamos que sí existe una causalidad y razonabilidad en el gasto porque sin el mismo, ante la inoperancia del Estado o tercero responsable, ante la falta del fluido eléctrico, la empresa no podrá continuar operando y, por ende, no estará en aptitud de producir renta. Es así que no puede negarse en el presente caso que la empresa pretende obtener una finalidad ulterior en reparar la hidroeléctrica, pues, de lo contrario, tendría que cerrar su

RESPONSABILIDAD

SOCIAL: ¿UN

REGALO O

UN GASTO

NECESARIO?

ENTRE EL BUEN

GOBIERNO

CORPORATIVOY LA

DETERMINACIÓN

DEL IMPUESTO A

LA RENTA

SOCIAL

RESPONSIBILITY:

A GIFT OR A

NECESSARY

EXPENSE?

BETWEEN GOOD

CORPORATE

GOVERNANCE

AND THE

DETERMINATION

OF INCOME TAX 
empresa al no poder operar debido a la falta de electricidad. Es claro que en el ejemplo planteado, este gasto se realiza con la finalidad de solicitar una recompensa: contar con el fluido eléctrico.

Este criterio ha sido cuestionado por el Tribunal Fiscal. A modo de ejemplo, señalamos la resolución del Tribunal Fiscal 013558-3-2009, donde se negó a la empresa la posibilidad de deducir el gasto, al considerar que se trataba de gastos que no eran indispensables y porque dichos gastos debían ser de cargo del Estado. En dicha jurisprudencia el Tribunal Fiscal precisa lo siguiente:

Se consideró no haberse acreditado el carácter de indispensable de la obra y que, además esta debió en todo caso ser de cargo del Estado. En consecuencia, se mantuvo el reparo a la deducción del aporte realizado en virtud de una adenda a un convenio suscrito por la recurrente con dos municipalidades y una empresa. Asimismo, se mantuvo el reparo a la deducción de una donación entregada a la Municipalidad Provincial de Islay para que esta a su vez cumpliera con un compromiso asumido con un tercero.

El Tribunal Fiscal ha establecido, en la misma línea (resoluciones 13558$3-2009,04807-1-2006$ y 0132-5-2004), que para que un gasto no sea considerado como una liberalidad, se consideran o agregan criterios como el de la extraordinariedad o el de la indispensabilidad y que no se encuentre a cargo de un tercero, para que pueda ser categorizado como deducible. Así, por ejemplo en el caso de los gastos realizados como consecuencia del trabajo de construcción de mejoramiento de carreteras, se debe cumplir con los siguientes criterios:

- Debe tener el carácter de extraordinario e indispensable para el transporte de los bienes que el titular de actividad minera produce desde o hasta su plantas y,

- no debe tratarse de una obra de infraestructura para beneficio de la comunidad, a cargo del Estado.

Pero el Poder Judicial tiene una posición contraria. Es así que tenemos la sentencia recaída en el proceso seguido contra el Tribunal Fiscal y SUNAT, expediente 2952-2008, demanda contencioso administrativa contra la resolución del Tribunal Fiscal 04807-1-2006. En esta sentencia la Sala señala como criterio para este tipo de gastos lo siguiente:

El Tribunal Fiscal confirmó los reparos por considerar que los gastos realizados no eran extraordinarios ni indispensables, y suponían más bien una actividad periódica para la conservación de la vía que correspondía ser efectuada por ProVias Nacional. 
Sin embargo los argumentos de la sentencia de la Sala Civil Transitoria fueron en esencia los siguientes:

El mantenimiento de carreteras no está comprendido dentro del concepto de infraestructura pública, por lo que para realizare dicha labor la empresa no necesitaba la aprobación de ningún organismo estatal.

No existe norma que establezca que el gasto, para ser causal, deba ser a la vez indispensable y obligatorio, por lo que no se trata de liberalidades sino de gastos necesarios para generar renta y normales según las circunstancias señaladas.

Las normas que estatuyen beneficios de carácter fiscal no deben interpretarse con el alcance más restringido que el texto admite, sino en forma tal que el propósito de la ley se cumpla de acuerdo a los principios de una razonable y discreta interpretación, lo que vale tanto como admitir que las exenciones tributarias pueden resultar del indudable propósito de la norma o de su necesaria implicancia, ya que en este caso si bien el organismo recaudador deja de percibir una parte del tributo, ello es consecuencia de una valoración del legislador en el ejercicio de la política fiscal que ha considerado más útil, desde el punto de vista económico y social, la promoción de determinadas actividades, en comparación con el sacrificio materializado en la menor percepción del impuesto.

Como se observa, la Sala es clara en distinguir entre si un gasto es causal y si es indispensable o extraordinario, no equipara dichos criterios. Por lo tanto, un gasto podría cumplir con el criterio de causalidad perfectamente y ser deducible sin que este sea indispensable o extraordinario, porque lo que resulta importante, para distinguir si un gasto es causal o no, es determinar qué beneficio tendrá la empresa, si repercutirá en la generación de la renta o en el mantenimiento de la misma. Es más, también la Sala establece el criterio, según el cual, inclusive cuando este gasto deba ser realizado por un tercero, como el Estado, ante la inoperancia del mismo (lo cual generaría problemas o no facilitaría el desempeño de la empresa), la empresa puede realizar el gasto, claro está, dejando establecida la relación causal respectiva.

Por lo expuesto, se infiere que el Tribunal Fiscal, conjuntamente con la Administración tributaria, ha considerado, en la mayoría de estos casos, como liberalidades a este tipo de gastos, salvo que acrediten que se tratan de gastos extraordinarios e indispensables que no deben de ser realizados por terceros. Esta postura es refutada por el Poder Judicial, motivo por el cual debemos considerar que para que este gasto sea deducible, debe cumplir con los criterios generales que todo gasto debe considerar para ser deducible: causalidad, proporcionalidad, razonabilidad y normalidad, sin otro requisito adicional como pretende el Tribunal Fiscal porque nuestra normativa no ha establecido dichos criterios como restrictivos

RESPONSABILIDAD

SOCIAL: ¿UN

REGALO O

UN GASTO

NECESARIO?

ENTRE EL BUEN

GOBIERNO

CORPORATIVOY LA

DETERMINACIÓN

DEL IMPUESTO A

LA RENTA

SOCIAL

RESPONSIBILITY:

A GIFT OR A

NECESSARY

EXPENSE?

BETWEEN GOOD

CORPORATE

GOVERNANCE

AND THE

DETERMINATION

OF INCOME TAX 
del gasto. Es así que en este caso, como todo gasto, adicionalmente de cumplir con los criterios antes señalados, debe encontrarse debidamente acreditado con los medios probatorios fehacientes, de modo tal que sea posible acreditar la causalidad y los demás requisitos establecidos para tal caso.

Un tema de mucha relevancia, no solo en este tipo de gasto, sino en un gasto en general, es el tema probatorio. Sobre este tema en específico — sustento del gasto de responsabilidad social — se han pronunciado Córdova Arce y Barrenechea Salazar. Ellos ofrecen las siguientes observaciones acerca de cuáles son los medios probatorios que pueden ayudar a sustentar este tipo de gastos:

La documentación sustentatoria especifica dependerá de cada caso concreto. No obstante en líneas generales sería conveniente contar con un acuerdo formal por convenio suscrito con las comunidades o entidades beneficiarias del gasto, en el que se detalle el compromiso asumido por la empresa frente a la comunidad y el propósito del mismo (promover el desarrollo de la comunidad, proteger el medio ambiente entre otros). Asimismo, sería conveniente contar con actas de los órganos societarios pertinentes que aprueben el gasto y sustenten la necesidad de su realización. La necesidad del gasto podría sustentarse a su vez con estudios que muestren el contexto social de las comunidades aledañas al proyecto, el historial de conflictos sociales que se hubiera presentado en el pasado y las necesidades y dificultades que se presentan en cada caso específico que deben ser subsanadas a efecto de viabilizar el desarrollo de cualquier proyecto de la industria extractiva. Adicionalmente, las empresas deberán tomar en consideración que los gastos de responsabilidad social deberán estar respaldados en los respetivos comprobantes de pago conforme a ley ${ }^{10}$.

Como puede observarse, en este caso se hace referencia a la jurisprudencia que desde hace ya varios atrás ha sentado el criterio que un gasto no solo debe ser sustentado con el comprobante de pago y el contrato, sino que debemos de contar con medios probatorios adicionales que permitan demostrar la causalidad del gasto realizado.

\section{CONCLUSIONES}

De lo expuesto, podemos concluir que para que estos gastos sean deducibles, deben acreditarse, con medios probatorios, la causalidad y necesidad de los mismos, principios ambos — sobre todo el primeroque al parecer han iniciado una variación en su correspondiente

10 Córdova ARCE, Alex y Lorena BARRENECHEA SALAZAR. «Impuesto a la renta y responsabilidad social». lus et Veritas, 46 (2013), p. 374. 
contenido conceptual recientemente. Esto ha ocurrido de manera tal que la diferencia entre causalidad y liberalidad podría devenir en una diferenciación muy tenue en determinados casos (tal como lo planteado al inicio de este artículo). Por ello y para poder establecer dicha diferencia, es importante efectuar un análisis exhaustivo y detallado de cada caso en particular. Por lo tanto, una primera conclusión sería indicar que, dado el reciente interés por parte de las empresas en realizar esta clase de gastos con motivo de la responsabilidad social, y a efectos de que el mismo sea causal, se debe aceptar verificar el cumplimiento de requisitos tales como: razonabilidad, proporcionalidad, usos y costumbres para el sector en donde se encuentra la empresa. Así, estos resultan ser criterios que establecen un primer marco general, pero que son insuficientes para determinar la deducibilidad de este tipo de gastos, porque este escaso marco regulatorio aplicable a esta figura no llega a cumplir dicho cometido.

En ese orden de ideas, consideramos que se debe evaluar caso por caso hasta que nuestro legislador, con un criterio que pueda recoger la realidad y no limitarla de manera indiscriminada, establezca algunos parámetros que puedan otorgar certeza a los contribuyentes, dado que en muchos casos la inversión o el egreso resulta un monto considerable, el cual, de ser reparado sin ningún criterio rector válido, puede en muchos casos limitar la actividad empresarial. Esto resulta especialmente problemático pues dicha actividad promueve la actividad económica en algunos lugares alejados del país, en donde lamentablemente el Estado no llega cumplir a cabalidad sus objetivos.

Otra conclusión que podríamos plantear es que restringir este tipo de gastos a situaciones donde el Estado no deba intervenir limita de manera injustificada y poco técnica este animo de establecer un ambiente adecuado y armonizado entre la empresa y su comunidad.

De lo señalado anteriormente cabe precisar que, aunque las posiciones esgrimidas nos permiten sustentar como deducible o no el gasto por responsabilidad social, ellas pueden resultar en posiciones extremas. La primera señala que estos gastos solo deben restringirse a eliminar posibles conflictos que impidan el normal desarrollo de la empresa y/o solucionarlos. La segunda, de manera extensa, comprende en general el gasto encaminado a armonizar el ambiente con la empresa. También es correcto señalar que estas posiciones no deben entenderse como una dicotomía, sino que la señalada segunda posición debe ser complementaria a la primera; de modo tal que ni la una sea tan restrictiva ni la otra tan extensiva. Esto permitiría además guardar el equilibrio a efectos de propender a la correcta aplicación del principio de causalidad, al demostrar que la inversión en el entorno social es necesaria, pese a no existir conflictos sociales, pues ella no es solo preventiva (al evitar la

RESPONSABILIDAD

SOCIAL: ¿UN

REGALO O

UN GASTO

NECESARIO?

ENTRE EL BUEN

GOBIERNO

CORPORATIVOY LA

DETERMINACIÓN

DEL IMPUESTO A

LA RENTA

SOCIAL

RESPONSIBILITY:

A GIFT OR A

NECESSARY

EXPENSE?

BETWEEN GOOD

CORPORATE

GOVERNANCE

AND THE

DETERMINATION

OF INCOME TAX 
ocurrencia de dichos conflictos), sino que genera un beneficio directo o indirecto también para la empresa, tal como es el caso, por ejemplo, del mejoramiento del ecosistema que rodea a la empresa, entre otros.

Finalmente, es importante resaltar el hecho de que el gasto por responsabilidad social, el derecho tributario guiado por una política fiscal desde un plano general, deba cumplir con el objetivo de señalar criterios y establecer pautas que nos otorguen no solo un adecuado marco jurídico tributario, sino también una necesaria e indispensable seguridad jurídica, aun cuando actualmente y según lo ya señalado, el análisis del cumplimiento del principio de causalidad de acuerdo con los lineamientos establecidos deba realizarse caso por caso. 